\title{
HYPERPARATHYROIDISM WITH INGREASED BONE DENSITY IN THE AREAS OF GROWTH
}

\author{
A. Adam and D. Ritchie, Aberdeen, Scotland
}

From the Orthopaedic and Radiodiagnostic Departments, Royal Infirmary, Aberdeen

A girl aged fourteen years complained of aching pain and swelling below the right knee for ten months. She was easily tired during this time, and had polydipsia. Three weeks before admission the leg gave way under her, with severe pain.

On examination, she was a healthy-looking, well developed girl. There was swelling over the right upper tibial shaft, which was tender on palpation. The underlying bone felt soft on pressure with the finger. Otherwise, general examination gave negative results.

Radiographic examination-The radiograph of the right knee (Fig. 1) showed a pathological fracture extending through a large multilocular cyst which expanded the proximal part of the tibial shaft and the metaphysis. The bone cortex overlying the cyst was of "egg-shell " thickness. A considerable degree of osteoporosis was present in the surrounding bone and, in addition, there were unusual bands of increased density occupying the juxta-epiphysial parts of femur, tibia and fibula.

A radiological survey of the skeleton was carried out. This confirmed the presence of generalised osteoporosis. In the long bones the cortex was thinned, at places assuming a pencil-line appearance, while the medullary cavity was correspondingly widened and the trabecular pattern accentuated. The skull showed a granular type of osteoporosis. Apart from the diminished bone density there was little abnormal in spine or pelvis.

Cyst formation was widespread throughout the long bones. The cysts were small, apart from the one described above.

The zones of increased density seen at the right knee were also found to be present in the metaphyses of all the long bones (Fig. 2). The density was a true increase as demonstrated against a control of the same sex and age (Fig. 3). Little bone structure was evident in these regions, which were of a rather amorphous appearance, and the bands were well defined where they merged with the adjoining bone. Strikingly, these bands were widest where bone growth was most active, especially at the knee and wrist. They were absent from the elbow where fusion of the epiphyses had occurred (Fig. 4).

The picture on the whole showed typical changes of hyperparathyroidism with the added unusual features of zones of increased density at the areas of growth.

Radiological examination of the neck and chest failed to demonstrate a parathyroid tumour.

Investigations-The serum calcium, on four estimations, varied from 18 to 18.5 milligrams per 100 cubic centimetres; the serum phosphorus was $\mathbf{2 . 6}$ milligrams; and the serum alkaline phosphatase was 52.5 units. Examination of the urine was negative, and renal function tests were within normal limits. Urinary calcium excretion averaged $\mathbf{0 . 6}$ grammes daily.

Treatment and progress-A diagnosis of primary hyperparathyroidism was made, and operation was performed by $\mathrm{Mr} \mathrm{G}$. G. Bruce. A small encapsulated tumour was found at the left lower pole of the thyroid. This was easily removed. It weighed 3 grammes and on section showed appearances typical of a parathyroid adenoma.

Convalescence was uneventful. The blood chemistry was normal on the day after operation and has remained so since. When last seen, seven months after operation, the patient was free from symptoms. 


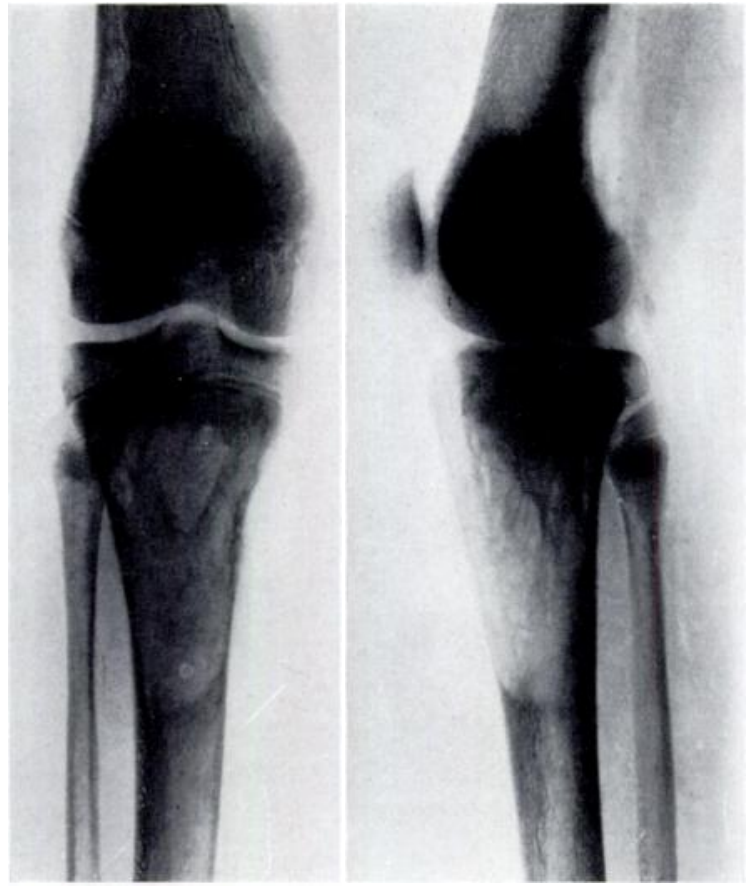

Fig. 1

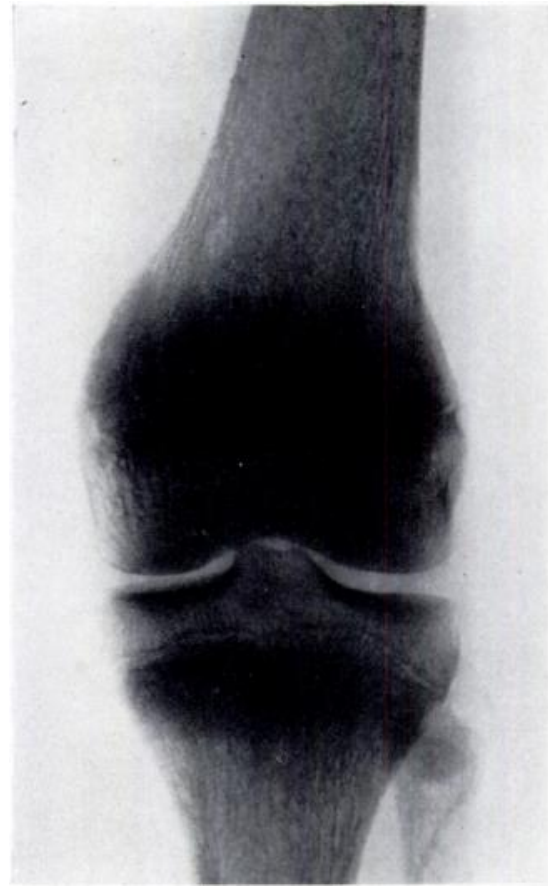

Fic. 2

Figure 1-Radiographs of right knee showing crst in upper tibia and dense bands in metaphyses of femur, tibia and fibula. Figure 2-Radiograph of left knee. Dense bands in metaphyses.

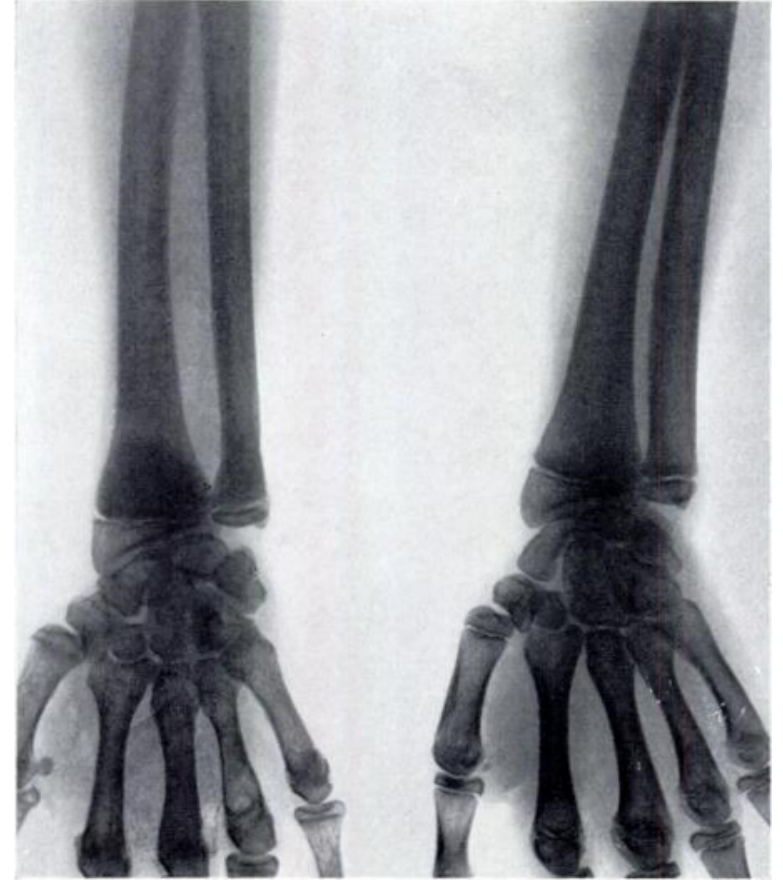

Fig. 3

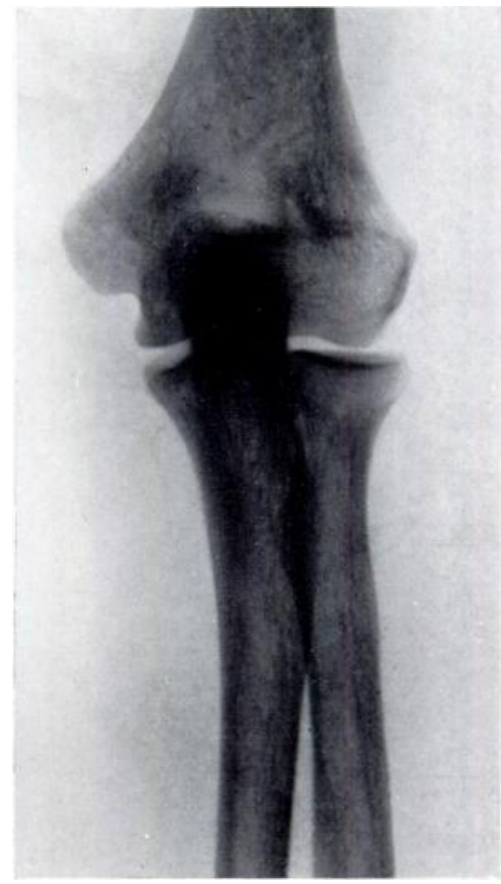

FIG. 4

Figure 3-Comparative radiograph of forearm with girl of same age. Figure 4-Radiograph of elbow showing fused epiphysis and absence of dense bands. 
Periodic radiographic examinations showed a striking improvement. The cyst at the right knee filled with new bone and the fracture united soundly (Fig. 5). Bone density generally returned to normal and the dense bands at the growing ends steadily became less obvious. At the knees the increased density is greatly diminished though it is not yet completely clear (Fig. 6). The latest radiographs of the wrists are now entirely normal (Fig. 7).

\section{DISCUSSION}

Primary hyperparathyroidism in children is rare. Of 3336 cases collected from the literature by Norris (1947), the patient was under fifteen years of age in seven. The classical features are like those found in adults, but in our case the finding of bands of increased density was unusual. In 1942 Aub presented to the Association for the Study of Internal

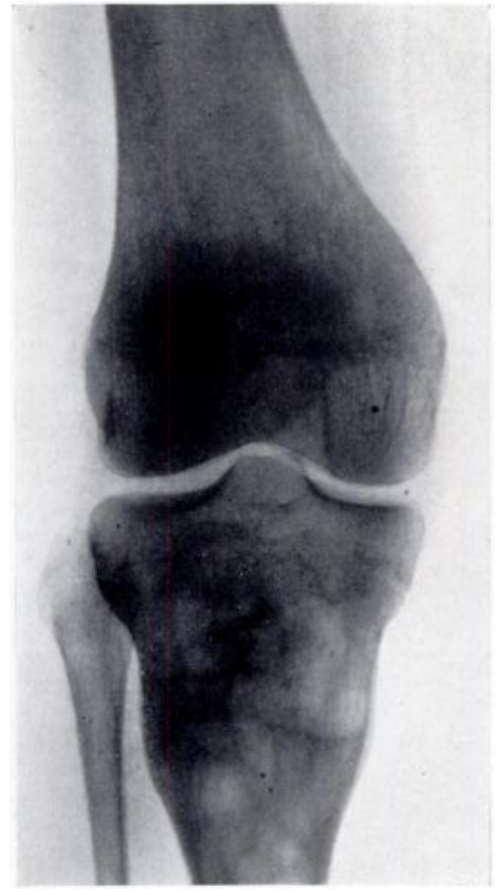

FIG. 5

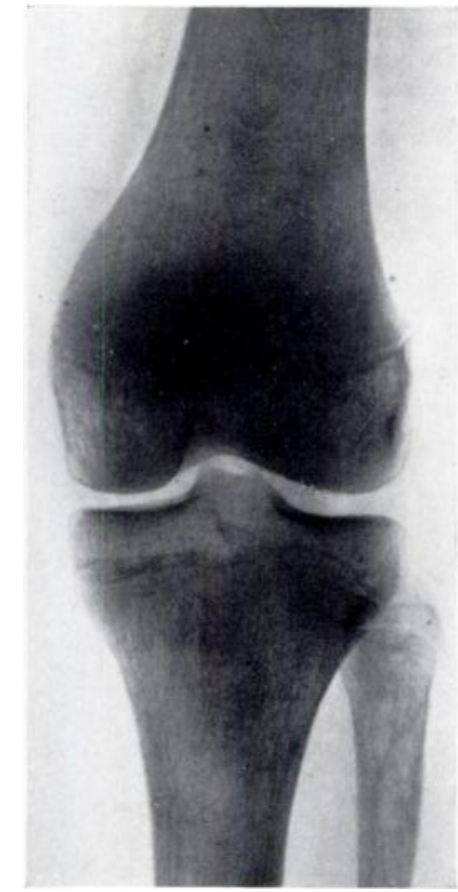

FIG. 6

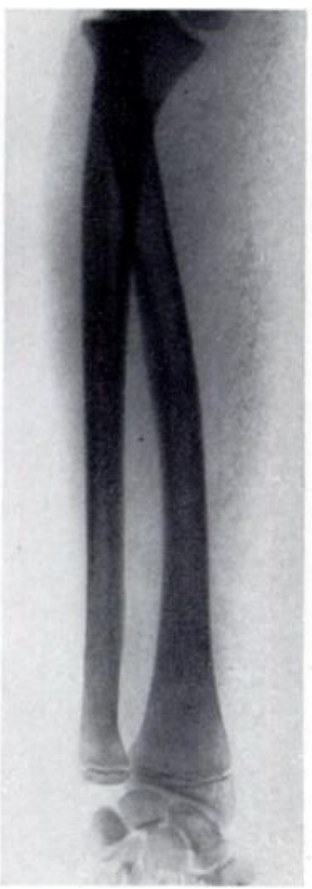

FIG. 7

Figure 5-Right knee seven months after operation. Figure 6-Left knee seven months after operation. Figure 7-Right forearm seven months after operation.

Secretions "a case of a parathyroid tumour in a growing boy, showing differentiation between decalcification of the shaft of the bone with great calcification in the areas of growth." In a personal communication he stated that the increased density was particularly striking about the knees, and that the changes disappeared in the year or so after operation. As far as we are aware this is the only reference in the literature to a similar case, but in two further cases published comparable dense bands can be seen in the radiographs reproduced (Pemberton and Geddie 1930, Fairbank 1951).

In other cases the relevant radiographs have not been published. Bearing this in mind, along with the fact that primary hyperparathyroidism in childhood is rare, it seems probable that these changes occur in a high proportion of cases and may even be the usual finding in the disease at this age.

Selye (1932) found that, in the later stages of experimental hyperparathyroidism in rats, formation of osteoclasts changed to proliferation of osteoblasts with formation of dense bone.

vol. $36 \mathrm{~B}$, No. 2 , MAY 1954 
This would indicate that osteoblasts are capable of laying down bone of increased density even in the presence of excess parathormone. It is suggested that this is what in fact takes place at the growing end of the bone.

\section{SUMMARY}

A case of parathyroid adenoma in a growing girl is described in which radiographs showed bands of increased density in the metaphyses in addition to the usual signs of osteitis fibrosa cystica. The literature is reviewed and the appearances are discussed.

\section{REFERENCES}

AuB, J. C. (1942): Presentation of a Case of a Parathyroid Tumor in a Growing Boy, Showing Differentiation between Decalcification of the Shaft of the Bone with Great Calcification in the Areas of Growth. Endocrinology, 30, S1,024.

AUB, J. C. (1953): Personal communication.

Fairbank, Sir T. (1951): An Atlas of General Affections of the Skeleton. Edinburgh: E. \& S. Livingstone Ltd. NorRis, E. H. (1947): The Parathyroid Adenoma. International Abstracts of Surgery, 84, 1.

Pemberton, J. de J., and Geddie, K. B. (1930): Hyperparathyroidism. Annals of Surgery, 92, 202.

Selye, H. (1932): On the Stimulation of New Bone-Formation with Parathyroid Extract and Irradiated Ergosterol. Endocrinology, 16, 547. 